\title{
Measurement of the vector analyzing power in elastic electron-proton scattering as a probe of the double virtual Compton amplitude
}

\author{
S. P. Wells, ${ }^{1}$ T. Averett, ${ }^{2}$ D. Barkhuff, ${ }^{3}$ D. H. Beck, ${ }^{4}$ E. J. Beise, ${ }^{5}$ C. Benson, ${ }^{4}$ H. Breuer, ${ }^{5}$ R. Carr, ${ }^{6}$ S. Covrig, ${ }^{6}$ \\ J. DelCorso, ${ }^{7, *}$ G. Dodson, ${ }^{3, \dagger}$ C. Eppstein, ${ }^{6}$ M. Farkhondeh, ${ }^{3}$ B. W. Filippone, ${ }^{6}$ T. A. Forest, ${ }^{4,}$ P. Frasier, ${ }^{6}$ R. Hasty, ${ }^{4}$ \\ T. M. Ito, ${ }^{6}$ C. Jones, ${ }^{6}$ W. Korsch, ${ }^{8}$ S. Kowalski, ${ }^{3}$ P. Lee, ${ }^{6}$ E. Maneva, ${ }^{6}$ K. McCarty, ${ }^{6}$ R. D. McKeown, ${ }^{6}$ J. Mikell, ${ }^{4}$ \\ B. Mueller, ${ }^{9}$ P. Naik, ${ }^{4}$ M. L. Pitt, ${ }^{7}$ J. Ritter, ${ }^{4}$ V. Savu, ${ }^{6}$ D. T. Spayde, ${ }^{5}$ M. Sullivan, ${ }^{6}$ R. Tieulent, ${ }^{5}$ E. Tsentalovich, ${ }^{3}$ \\ B. Yang, ${ }^{3}$ and T. Zwart ${ }^{3}$ \\ ${ }^{1}$ Center for Applied Physics Studies, Louisiana Tech University, Ruston, Louisiana 71272 \\ ${ }^{2}$ Department of Physics, College of William and Mary, Williamsburg, Virginia 23187 \\ ${ }^{3}$ Bates Linear Accelerator Center, Laboratory for Nuclear Science and Department of Physics, \\ Massachusetts Institute of Technology, Cambridge, Massachusetts 02139 \\ ${ }^{4}$ University of Illinois at Urbana-Champaign, Urbana, Illinois 61801 \\ ${ }^{5}$ University of Maryland, College Park, Maryland 20742 \\ ${ }^{6}$ Kellogg Radiation Laboratory, California Institute of Technology, Pasadena, California 91125 \\ ${ }^{7}$ Department of Physics, Virginia Polytechnic Institute and State University, Blacksburg, Virginia 24061 \\ ${ }^{8}$ Department of Physics and Astronomy, University of Kentucky, Lexington, Kentucky 40506 \\ ${ }^{9}$ Physics Division, Argonne National Laboratory, Argonne, Illinois 60439
}

(Received 22 February 2000; published 1 May 2001)

\begin{abstract}
We report the first measurement of the vector analyzing power in inclusive transversely polarized elastic electron-proton scattering at $Q^{2}=0.1(\mathrm{GeV} / c)^{2}$ and large scattering angles. This quantity must vanish in the single virtual photon exchange, plane-wave impulse approximation for this reaction, and can therefore provide information on two photon exchange amplitudes for electromagnetic interactions with hadronic systems. The observable we have measured is driven by the imaginary part of the two photon exchange amplitude, the hadronic side of which is simply the Compton amplitude for the proton with two virtual photons. We find a small but nonzero value of $A=-15.4 \pm 5.4 \mathrm{ppm}$.
\end{abstract}

DOI: 10.1103/PhysRevC.63.064001

PACS number(s): 24.70.+s, 13.40.-f, 13.60.Fz, 14.20.Dh

The recent development and refinement of experimental methods for measurements of small (few parts per million, or ppm) parity violating effects in polarized electron scattering [1-3] provides a new technique for further studies of the electromagnetic structure of the proton. We have exploited these methods for the first time to measure the small vector analyzing power in the elastic scattering of $200 \mathrm{MeV}$ electrons from the proton at large laboratory scattering angles $\left(130^{\circ} \leqslant \theta \leqslant 170^{\circ}\right.$ ), corresponding to a four-momentum transfer squared of $Q^{2}=0.1(\mathrm{GeV} / c)^{2}$. This parity conserving quantity is associated with transverse electron polarization, in contrast to the parity violating longitudinal (i.e., helicitydependent) asymmetry. It has been previously noted [4] that transverse polarization effects will be suppressed by the relativistic boost factor $1 / \gamma$. Nevertheless, as demonstrated here, the development of the technology to measure small parity violating asymmetries, along with the ability to produce transversely polarized electron beams at high energies, now renders these transverse polarization effects amenable to measurement.

\footnotetext{
*Present address: Department of Physics, College of William and Mary, Williamsburg, VA 23187.

${ }^{\dagger}$ Present address: Spallation Neutron Source, Oak Ridge National Laboratory, Oak Ridge, TN 37830-8218.

* Present address: Center for Applied Physics Studies, Louisiana Tech University, Ruston, LA 71272.
}

The vector analyzing power is a time-reversal odd observable that must vanish in first-order perturbation theory, and can only arise in leading order from the interference of twophoton exchange (second order) and single-photon exchange amplitudes. Our observation of this quantity therefore demonstrates the viability of a new technique to access the physics associated with the absorption of two virtual photons by a hadronic system. Thus, the study of vector analyzing powers provides another method to study processes in which two photons couple to the proton, i.e., the Compton amplitude, that is complementary to virtual Compton scattering (VCS), in which there is presently a great deal of interest as a means to further probe the structure of the proton [5]. VCS involves the coupling of one virtual and one real photon to a hadronic system, but in practice includes Bethe-Heitler amplitudes, associated with radiation of a real photon from the electron, with which care must be taken for proper treatment to allow a correct interpretation of those measurements. In contrast, the two-photon exchange amplitude involves the coupling of two virtual photons to a hadronic system, and the vector analyzing power in elastic electron-proton scattering, which cannot include a background from Bethe-Heitler amplitudes, potentially offers an attractive alternative to access this fundamental amplitude.

As will be shown below, the vector analyzing power in elastic electron-proton scattering directly probes the imaginary part of the double virtual Compton amplitude, which can be related through dispersion integrals [6] to the real part of this amplitude, in which the generalized polarizabilities of 


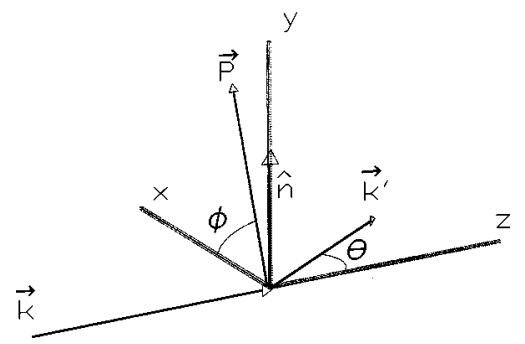

FIG. 1. Coordinate system showing the orientations of the incident and scattered electron wave vectors $\mathbf{k}$ and $\mathbf{k}^{\prime}$, respectively, the unit vector $\hat{\mathbf{n}}$, the transverse polarization vector $\mathbf{P}$, and the angles $\theta$ and $\phi$.

the nucleon are contained. It was also noted in [6] that the Compton amplitude has increased sensitivity to the generalized polarizabilities above the pion threshold, and that the dispersion analysis developed allows for an extension of our knowledge of these nucleon structure-dependent quantities. The kinematics of the measurements reported here, i.e., a beam energy of $200 \mathrm{MeV}$, which is just above the pion threshold, provide for a measurement of the imaginary part of the Compton amplitude above pion threshold, from which an extension of the generalized polarizabilities can be obtained through such a dispersion analysis.

The vector analyzing power in electron-nucleus scattering results in a spin-dependent asymmetry, which can, for example, be generated by the interaction of the electron spin with the magnetic field seen by the electron in its rest frame [7]. This spin dependence in the scattering cross section $\sigma(\theta)$ can be written as $[8,9]$

$$
\sigma(\theta)=\sigma_{0}(\theta)\left[1+A_{n}(\theta) \mathbf{P} \cdot \hat{\mathbf{n}}\right],
$$

where $\sigma_{0}(\theta)$ is the spin-averaged scattering cross section and $A_{n}(\theta)$ is the vector analyzing power for the reaction for the electron beam polarization vector $\mathbf{P}$ (which is proportional to the spin vector operator $\mathbf{S}$ ) oriented along the $\hat{\mathbf{n}}$ axis. As shown in Fig. 1, the unit vector $\hat{\mathbf{n}}$ is normal to the scattering plane, and is defined through $\hat{\mathbf{n}} \equiv\left(\mathbf{k} \times \mathbf{k}^{\prime}\right) / \mid \mathbf{k}$ $\times \mathbf{k}^{\prime}$, where $\mathbf{k}$ and $\mathbf{k}^{\prime}$ are wave vectors for the incident and scattered electrons, respectively. The scattering angle $\theta$ is found through $\cos \theta=\left(\mathbf{k} \cdot \mathbf{k}^{\prime}\right) /|\mathbf{k}|\left|\mathbf{k}^{\prime}\right|$, and, in the Madison convention, is positive for the electron scattering to beam left for $\hat{\mathbf{n}}$ along the vertical (as in Fig. 1). The beam polarization $\mathbf{P}$ can be expressed in terms of the number of beam electrons with spins parallel $\left(m_{s}=+\frac{1}{2}\right)$ and antiparallel $\left(m_{s}=-\frac{1}{2}\right)$ to $\hat{\mathbf{n}}$, so that the measured asymmetry at a given scattering angle $\epsilon(\theta)$ in the plane to which $\hat{\mathbf{n}}$ is normal is defined through

$$
\epsilon(\theta)=\frac{\sigma_{\uparrow}(\theta)-\sigma_{\downarrow}(\theta)}{\sigma_{\uparrow}(\theta)+\sigma_{\downarrow}(\theta)}=A_{n}(\theta)\left\langle P_{n}\right\rangle,
$$

where $\sigma_{\uparrow, \downarrow}(\theta)$ is the differential cross section for $m_{s}=+\frac{1}{2}$ and $-\frac{1}{2}$, respectively. Thus, with knowledge of the magnitude of the incident beam polarization $\left\langle P_{n}\right\rangle$ along the $\hat{\mathbf{n}}$ axis, measurement of $\epsilon(\theta)$ can yield a determination of the vector

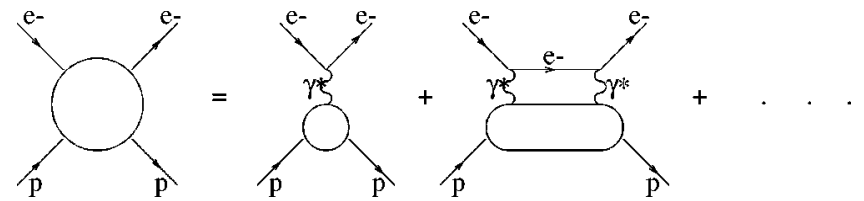

FIG. 2. Expansion of the electron-proton scattering amplitude in terms of Feynman diagrams through second order for one-photon exchange and two-photon exchange amplitudes.

analyzing power $A_{n}(\theta)$, which contains the underlying physics of the electron-proton interaction.

The vector analyzing power $A_{n}(\theta)$ for transverse electron beam polarization along the $\hat{\mathbf{n}}$ axis is defined in a modelindependent way through

$$
A_{n}(\theta)=\frac{\operatorname{Tr}\left\{M(\theta) \Sigma_{n} M^{\dagger}(\theta)\right\}}{\operatorname{Tr}\left\{M(\theta) M^{\dagger}(\theta)\right\}},
$$

where $M$ is the full amplitude for the electron-proton interaction and $\Sigma_{n}$ is a covariant spin operator for the incident electron polarized along the $\hat{\mathbf{n}}$ axis. A perturbative expansion of the amplitude $M$ through second order gives

$$
M=M_{B}+M_{2 \gamma}+\cdots,
$$

where $M_{B}$ is the Born amplitude for the exchange of one virtual photon and $M_{2 \gamma}$ is the two-photon exchange amplitude, as shown diagrammatically in Fig. 2. Upon carrying through the spin algebra and traces of Eq. (3), and exploiting the fact that $M_{B}$ is purely real, one obtains [10]

$$
A_{n}=\frac{2 M_{B} \operatorname{Im}\left\{M_{2 \gamma}\right\}}{\left|M_{B}\right|^{2}}
$$

to leading order. Thus, the vector analyzing power in elastic electron-proton scattering is driven by the imaginary part of the two-photon exchange amplitude, the hadronic side of which is simply the double virtual Compton tensor.

Although here we do not explicitly evaluate the twophoton exchange amplitude, we note that there are calculations [10] of the vector analyzing power in electron proton elastic scattering underway, where the two photon exchange amplitude is expressed as a diagrammatic expansion as shown in Fig. 3. In the first term of this expansion, free proton form factors are used at the photon-proton vertices, and a free proton propagator is assumed in the intermediate proton state. Higher-order terms in this expansion include intermediate state resonances (e.g., the $\Delta^{+}$) and the creation of intermediate state virtual mesons.

Previous calculations of vector analyzing powers for low energy, spin- $\frac{1}{2}$ probes incident on heavy nuclei have been performed using the original derivation of Mott [11], where the energies are low enough to assume that the nucleus is simply a point charge of magnitude $Z e$. The analyzing powers calculated in these cases are much larger than the vector analyzing power for electron-proton scattering considered here, and this is commonly exploited as a means to measure the polarization of low-energy electron beams $(\sim 100 \mathrm{keV})$ 

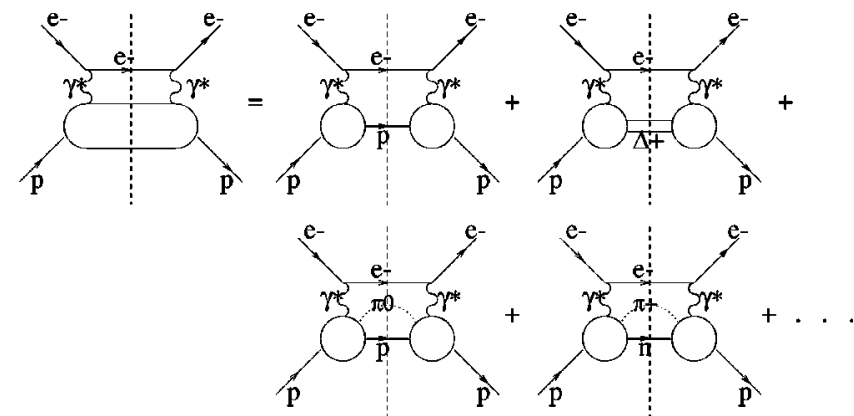

FIG. 3. Diagrammatic expansion of the two-photon exchange amplitude in terms of the number of allowed intermediate states for the hadronic system.

with the use of "Mott" polarimeters [7]. Such measurements, however, are not sensitive to the internal structure or spin of the hadronic system. More recently, some level of nuclear structure has been taken into account in calculations of analyzing powers for high-energy elastic scattering of transversely polarized electrons from heavy spin 0 nuclei at forward electron scattering angles, performed in the eikonal expansion and using finite charge densities for the nuclei [12]. In these calculations, the nonzero analyzing powers were generated through the distortion of the electron waves in the Coulomb potential of the nuclear targets, providing the needed extension beyond single-photon exchange to the distorted-wave impulse approximation. In the SAMPLE kinematics, the electron energy of $200 \mathrm{MeV}$ is much larger than the energies used for Mott polarimetry, the proton target has the smallest possible $Z$ so that Coulomb effects are at a minimum, and the electrons are scattered at large angles where magnetic effects are important. These facts, along with the spin- $-\frac{1}{2}$ nature of the proton, imply that our measurement of the vector analyzing power will be sensitive to nontrivial structure of the proton not taken into account in these previous theoretical treatments. Presently underway are calculations using the formalism outlined above in terms of the two-photon exchange amplitude as an expansion as shown in Fig. 3. It is these latter calculations to which we will compare our results below.

Using the apparatus for the SAMPLE experiment $[1,13]$, a high statistics measurement of the parity violating asymmetry in inclusive elastic $p\left(\vec{e}, e^{\prime}\right)$ scattering at the MIT/Bates Linear Accelerator Center, we have made measurements of the asymmetry in the elastic scattering of $200 \mathrm{MeV}$ transversely polarized electrons from the proton at backward scattering angles. This represents the first measurement of a vector analyzing power in polarized electron scattering from the proton at this high a momentum transfer. The data we report are the result of an experiment using a $200 \mathrm{MeV}$ polarized electron beam of average current $40 \mu \mathrm{A}$ incident on a $40 \mathrm{~cm}$ liquid hydrogen target [14]. The scattered electrons were detected in a large solid angle $(\sim 1.5 \mathrm{sr})$, axially symmetric air Cerenkov detector consisting of 10 mirrors, each shaped to focus the Cerenkov light onto one of ten shielded photomultiplier tubes. This combination of large solid angle and high luminosity allows measurements of small asymmetries in a relatively short period of time. The data presented here were acquired in just two days of running under these conditions. Properties of the detector signals and beam have been described in detail in Refs. [1,13], along with the method of asymmetry extraction and correction. Thus, here we report only the differences between the experimental running conditions for longitudinally polarized beams as used for parity violation measurements and the transversely polarized beam used for the vector analyzing power measurements. The systematic errors associated with the asymmetries from each of the individual mirrors are the same for these measurements as for those in Refs. [1,13], totaling 0.7 ppm, and are negligible compared with the overall statistical error of $5.4 \mathrm{ppm}$ obtained for these measurements.

The polarized laser light used on the bulk GaAs source crystal produces electron beams with longitudinal polarization, consequently significant spin manipulation was required to orient the beam polarization transversely. This was achieved with a Wien filter, which contains electric and magnetic fields oriented perpendicular to each other and to the beam direction, and a set of beam solenoids. The Wien filter was positioned immediately downstream of the source anode, and was used to precess the electron spin away from the beam direction $\left(\sim 90^{\circ}\right.$ for these measurements). The beam solenoids were positioned near the first accelerating cavity in the beam line, and precessed the resulting transverse components of the beam polarization. The combination of these beam line elements allowed the polarization direction to be chosen arbitrarily, and each element was calibrated such that the polarization direction is determined to $\pm 2^{\circ}$ [15].

For the measurements reported here, two orthogonal transverse beam polarizations were used during two running periods: one with the polarization directed to beam right (which we denote $\Phi=0$ ) and one with the polarization pointing up $(\Phi=90)$. The magnitude of the beam polarization was measured with a Moller apparatus positioned on the beam line, and averaged $36.3 \pm 1.8 \%$ during these measurements. Finally, to minimize false asymmetries and test for systematic errors, the electron beam polarization was manually reversed relative to all electronic signals, for both $\Phi$ $=0$ and $\Phi=90$ running, with the insertion of a $\lambda / 2$ plate in the laser beam. Thus, four separate sets of measurements were made: $\Phi=0, \lambda / 2$ IN and OUT; and $\Phi=90, \lambda / 2$ IN and OUT.

The elastic scattering transverse asymmetry was determined for each of the ten individual mirrors in each running configuration after correction for all effects, including beam polarization, background dilution, and radiative effects, as described in Refs. [1,13]. Although the geometry of this detector allowed for combining the asymmetries from individual mirrors positioned on opposite sides of the incident beam [via Eq. (2) and imposing the rotational invariance criterion $A(\theta)=-A(-\theta)$ [9]], we chose an alternative form of analysis wherein the full statistical information contained in the data set could be used to extract the vector analyzing power. Because the individual mirrors were positioned at varying azimuthal angles $\phi$ relative to the polarization direction (see Fig. 1), the asymmetries measured in the 
TABLE I. Polar $(\theta)$ and azimuthal $(\phi)$ angles of each individual mirror within the SAMPLE detector.

\begin{tabular}{rcc}
\hline \hline Mirror & $\theta(\mathrm{deg})$ & $\phi(\mathrm{deg})$ \\
\hline 1 & 146 & 135 \\
2 & 154 & 90 \\
3 & 146 & 45 \\
4 & 138 & 180 \\
5 & 161 & 180 \\
6 & 161 & 0 \\
7 & 138 & 0 \\
8 & 146 & 225 \\
9 & 154 & 270 \\
10 & 146 & 315 \\
\hline \hline
\end{tabular}

mirrors should follow a sinusoidal dependence in this angle. The sinusoidal dependence in the azimuthal angle $\phi$ is seen by rewriting Eq. (2) as

$$
\epsilon(\theta, \phi)=A_{n}(\theta) P \sin (\phi+\delta),
$$

where $\phi$ measures the angle of the polarization vector in the plane transverse to the beam direction, and the phase $\delta$ takes into account the direction of $\mathbf{P}$ relative to $\hat{\mathbf{n}}$. Table I summarizes the polar $(\theta)$ and azimuthal $(\phi)$ angles at the center of each individual mirror within the SAMPLE detector. As seen in Table I, mirrors 4 and 5 have the same azimuthal angle relative to the polarization direction, but different polar angles relative to the incident beam direction. A separate analysis, however, indicated that the polar angle dependence to the asymmetry was negligible, allowing us to combine the asymmetries from these two mirrors (similarly for mirrors 6 and 7) into one asymmetry at the same azimuthal angle $\phi$.

The data set for each $\Phi$ and $\lambda / 2$ running configuration therefore consists of eight data points at varying $\phi$ values, to which we perform a $\chi^{2}$ minimization to a two-parameter function via

$$
\chi_{\text {d.o.f. }}^{2}=\frac{1}{6} \sum_{i=1}^{8}\left[A_{n}^{i}-\left(a \sin \phi_{i}+b \cos \phi_{i}\right)\right]^{2} /\left[\delta A_{n}^{i}\right]^{2},
$$

which is linear in the coefficients $a$ and $b$. Here $A_{n}^{i}$ and $\delta A_{n}^{i}$ are the measured asymmetry and error, respectively, at each azimuthal angle $\phi_{i}$, corrected for all effects [1,13], including beam polarization normalization [as suggested in Eq. (6)]. The coefficients $a$ and $b$ can then be converted into an amplitude and phase, i.e.,

$$
A_{\text {fit }}=\left|A_{n}\right| \sin (\phi+\delta)
$$

as in Eq. (6), where the amplitude $\left|A_{n}\right|$ gives the magnitude of the vector analyzing power, and the phase $\delta$ verifies the direction of the beam polarization and determines the overall sign of the analyzing power.

The sinusoidal dependence just discussed is illustrated in Fig. 4, where the combined data for $\Phi=0$ and $\Phi=90$ are shown as a function of azimuthal angle, along with the best fit to the data according to the procedure outlined above.

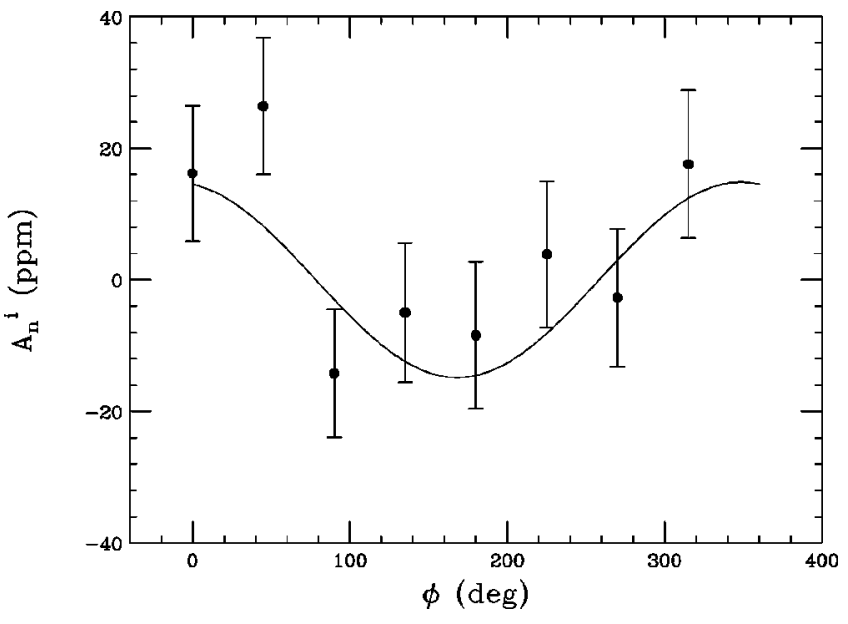

FIG. 4. Plot of the measured asymmetry, corrected for all effects including beam polarization, background dilution, and radiative effects, as a function of azimuthal scattering angle $\phi$ for the combined data of all four running configurations as described in the text. The curve represents the best fit to the data according to Eq. (8).

Here we have defined $\phi=0$ to be at beam left, and have taken into account the $90^{\circ}$ phase difference between the $\Phi$ $=0$ and $\Phi=90$ polarization directions. For these combined data, the overall $\chi^{2}$ per degree of freedom for the best fit was found to be 0.9 , providing a $50 \%$ confidence level that the data follow this dependence [16]. This should be compared, however, with the $\chi^{2}$ per degree of freedom of 2.1 for a fit to $A=0$, which has a corresponding confidence level of $4 \%$ that the data are consistent with $A=0$. Even if we allow an overall offset to a constant dependence, we find an average of $A=3.5 \pm 3.7 \mathrm{ppm}$, with a $\chi^{2}$ per degree of freedom of 1.9 , and a corresponding confidence level of $7 \%$.

In Table II, we summarize our results using this analysis procedure for the four independent running conditions. Note that the deduced magnitudes are all consistent within experimental errors, and the deduced phase changes by $180^{\circ}$ upon the insertion or removal of the $\lambda / 2$ plate as expected, and by $90^{\circ}$ from one $\Phi$ running configuration to the other. Combining these four independent measurements, we quote our final result: a vector analyzing power for elastic electron-proton scattering of $-15.4 \pm 5.4 \mathrm{ppm}$ at the average electron laboratory scattering angle of $146.1^{\circ}$, corresponding to $Q^{2}$ $=0.1(\mathrm{GeV} / \mathrm{c})^{2}$. To demonstrate the precision to which this quantity has been determined relative to the expected size of this effect, we plot this data point in Fig. 5 along with the prediction of Ref. [10] for the vector analyzing power for elastic electron-proton scattering. This calculation only in-

TABLE II. Results of the fitting procedure described in the text.

\begin{tabular}{cccrc}
\hline \hline$\Phi$ & $\lambda / 2$ & $\left|A_{n}\right|(\mathrm{ppm})$ & \multicolumn{1}{c}{$\delta(\mathrm{deg})$} & $\chi_{\text {d.o.f. }}^{2}$ \\
\hline 0 & IN & $12.9 \pm 9.8$ & $173.8 \pm 39.5$ & 1.30 \\
0 & OUT & $13.8 \pm 9.9$ & $16.9 \pm 39.5$ & 1.50 \\
90 & IN & $18.4 \pm 11.8$ & $-84.1 \pm 39.8$ & 0.30 \\
90 & OUT & $18.1 \pm 11.7$ & $127.2 \pm 38.0$ & 2.07 \\
\hline \hline
\end{tabular}




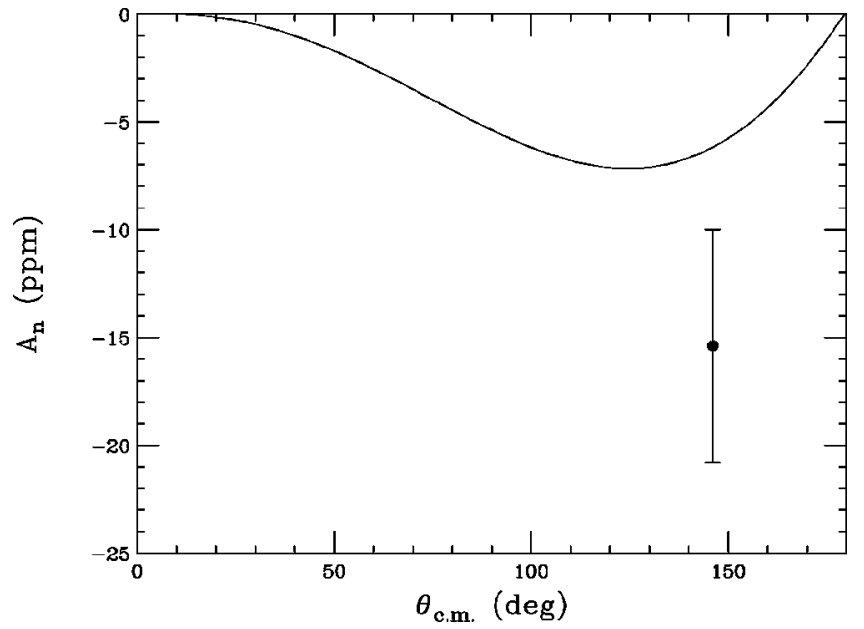

FIG. 5. Plot of the measured vector analyzing power in elastic electron-proton scattering in the SAMPLE kinematics as a function of center-of-mass scattering angle, along with the prediction of Ref. [10]. Details of the calculation are given in the text.

cludes free proton form factors at the virtual photon-proton vertices and a free proton propagator for the intermediate proton state to evaluate the two-photon exchange amplitude $M_{2 \gamma}$, i.e., the first term in the diagrammatic expansion of Fig. 3.
The data reported here represent the first measurement of a vector analyzing power in polarized electron scattering at this high a momentum transfer. Our observation of this quantity demonstrates the viability of a new technique to access physics associated with the Compton amplitude. We have also made measurements of the vector analyzing power in inclusive quasielastic electron-deuteron scattering, the results of which will be reported in a future paper. Further parity violation measurements at higher $Q^{2}$ values are planned [17] from both hydrogen and deuterium targets, where high statistics transverse asymmetry data will also be taken. These data, along with the first measurement reported here, will provide valuable information about the imaginary part of the Compton amplitude at relatively low beam energies, from which we can extend our knowledge of the nucleon generalized polarizabilities.

The skillful efforts of the staff at MIT/Bates to provide the high-quality beam required for these measurements, and useful conversations with T.W. Donnelly, A. Afanasev, and M. Rekalo, are gratefully acknowledged. We also thank A. Afanasev for providing us with the calculations. This work was supported by NSF Grant Nos. PHY-9870278 (Louisiana Tech), PHY-9420470 (Caltech), PHY-9420787 (Illinois), PHY-9457906/PHY-9229690 (Maryland), PHY-9733773 (VPI), and U.S. DOE Cooperative Agreement No. DE-FC0294ER40818 (MIT/Bates) and Contract No. W-31-109ENG-38 (ANL).
[1] D.T. Spayde et al., SAMPLE Collaboration, Phys. Rev. Lett. 84, 1106 (2000).

[2] K.A. Aniol et al., HAPPEX Collaboration, Phys. Rev. Lett. 82, 1096 (1999).

[3] R.D. McKeown, in Parity Violation in Atoms and Polarized Electron Scattering, edited by B. Frois and M.-A. Bouchiat (World Scientific, Singapore, 1999), p. 423.

[4] T.W. Donnelly and A.S. Raskin, Ann. Phys. (N.Y.) 169, 247 (1986).

[5] P.A.M. Guichon and M. Vanderhaeghen, Prog. Part. Nucl. Phys. 41, 125 (1998).

[6] B. Pasquini, D. Drechsel, M. Gorchtein, A. Metz, and M. Vanderhaeghen, hep-ph/0007144.

[7] T.J. Gay and F.B. Dunning, Rev. Sci. Instrum. 63, 1635 (1992), and references therein.
[8] J. Kessler, Polarized Electrons, 2nd ed. (Springer, Berlin, 1985).

[9] G.G. Ohlsen, Rep. Prog. Phys. 35, 717 (1972).

[10] A. Afanasev (private communication).

[11] N.F. Mott, Proc. R. Soc. London, Ser. A 135, 429 (1932).

[12] E.L. Kronenberg, Ph.D. thesis, MIT, 1989 (unpublished).

[13] B. Mueller et al., Phys. Rev. Lett. 78, 3824 (1997).

[14] E.J. Beise et al., Nucl. Instrum. Methods Phys. Res. A 378, 383 (1996).

[15] M. Pitt et al., in Proceedings of the 12th International Symposium on High-Energy Spin Physics (SPIN96), edited by C.W. de Jager et al. (World Scientific, Singapore, 1997), p. 813.

[16] Particle Data Group, L. Montanet et al., Phys. Rev. D 50, 1173 (1994).

[17] JLab Experiment E91-017, “'G0,” D. Beck, spokesperson. 